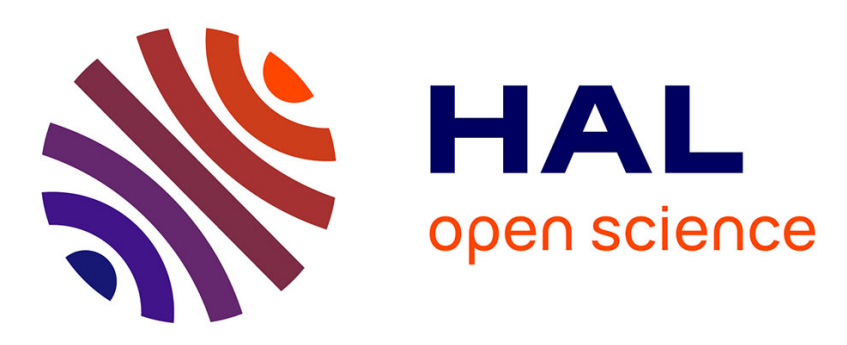

\title{
Stopping of a solid object in an elasto viscoplastic material
}

Dominique Tarlet, Eliane Younes, Stéphane Roux, Arthur Levy, Teodor Burghelea

\section{- To cite this version:}

Dominique Tarlet, Eliane Younes, Stéphane Roux, Arthur Levy, Teodor Burghelea. Stopping of a solid object in an elasto viscoplastic material. Journal of Non-Newtonian Fluid Mechanics, 2019, 263, pp.120-129. 10.1016/j.jnnfm.2018.11.006 . hal-02358756

\section{HAL Id: hal-02358756 https://hal.science/hal-02358756}

Submitted on 26 Nov 2020

HAL is a multi-disciplinary open access archive for the deposit and dissemination of scientific research documents, whether they are published or not. The documents may come from teaching and research institutions in France or abroad, or from public or private research centers.
L'archive ouverte pluridisciplinaire HAL, est destinée au dépôt et à la diffusion de documents scientifiques de niveau recherche, publiés ou non, émanant des établissements d'enseignement et de recherche français ou étrangers, des laboratoires publics ou privés. 


\title{
Stopping of a solid object in an elasto viscoplastic material
}

\author{
Dominique Tarlet, Eliane Younes, Stéphane Roux, Arthur Levy, Teodor Burghelea* \\ Université de Nantes, Nantes Atlantique Universités, Laboratoire de Thermique et Energie de Nantes, UMR 6607, CNRS, La Chantrerie, Rue Christian Pauc, B.P. 50609, \\ F-44306 Nantes Cedex 3, France
}

\begin{abstract}
A B S T R A C T
The impact and stopping of a spherical object freely falling onto the surface of an elasto-viscoplastic material (an aqueous solution of Carbopol ${ }^{\circledR}$ Ultrez 10) is studied both experimentally and theoretically. Accurate measurements of the instantaneous position of the centre of mass of the falling object allow one to calculate both the speed and acceleration of the object. To rationalise the experimental results we resort to a simple $1-D$ toy model initially proposed by Putz and Burghelea [1]. At early stages of the motion, the deformation of the gel is dominated by viscous effects, the model by Putz and Burghelea reduces to the well-known Herschel-Bulkley relationship and an equation of motion for the sphere can be derived. By comparing the measured trajectories, speeds and accelerations with the "viscous" analytical solution one may estimate the yield stress, the consistency and the power law index. After long enough times, the spherical object oscillates around its final stopping position and the deformation of the material is no longer viscous but elastic. Within this second asymptotic limit the model by Putz and Burghelea reduces to the Hooke's law and once more an analytical solution for the equation of motion may be readily obtained. By measuring the period of the oscillatory motion observed during this regime one may estimate the elastic modulus of the gel.
\end{abstract}

\section{Introduction}

A broad class of materials exhibit a dual response when subjected to an external stress. For low applied stresses they behave as solids (loosely speaking they may deform but they do not flow) but, if the stress exceeds a critical threshold generally referred to as the "yield stress", they behave as fluids (typically non-Newtonian) and a macroscopic flow is observed. This distinct class of materials has been termed as "yield stress materials" and, during the past several decades it attracted a constantly increasing level of interest from both theoreticians and experimentalists. From a fundamental standpoint, yield stress materials continue triggering intensive debates and posing difficult challenges to both theoreticians and experimentalists from various communities: soft matter physics, rheology, physical chemistry and applied mathematics. The progress in understanding the flow behavior of yield stress materials made the object of several recent review papers [2-5]. The best known debate concerning the yield stress materials is undoubtedly that related to the very existence of a "true" yield stress behavior [6,7]. During the past two decades, however, a number of technical improvements of the rheometric equipment made possible measurements of torques as small as $0.1 \mathrm{n} \mathrm{Nm}$ and of rates of deformation as small as $10^{-7} \mathrm{~s}^{-1}$. Such accurate rheological measurements proved unequivocally the existence of a true yielding behavior $[1,8,9]$. The yielding transition of an elasto-viscoplastic material remains, however, elusive. It has been recently shown that, even in the case a "simple" yield stress fluid, the transition from a solid like regime described by the Hooke's law $\sigma=G \gamma$ to a viscous regime is not direct but mediated by an intermediate regime were the elastic and viscous effects coexist, [1]. Here $\sigma$ stands for the applied stress, $G$ for the elastic modulus and $\gamma$ for the deformation of the material.

The viscous deformation regime has been classically described by the Herschel-Bulkley model $[10,11]$ :

$\sigma=\sigma_{y}+K \dot{\gamma}^{n}$

where $\sigma_{y}$ is the yield stress, $K$ the consistency and $n$ the power law index. This correlation is typically used to measure the yield stress $\sigma_{y}$ by fitting flow curves.

A minimal rheological characterisation of an elasto - viscoplastic material requires two types of rheological tests to be performed: small amplitude oscillatory tests for the assessment of the elastic moduli and flow ramps performed in either a controlled stress or a controlled rate of strain mode for the assessment of the yield stress, consistency and power law index. In spite of an increased instrumental accuracy of the modern rotational rheometers, the instrumental error of the measurements of the rheological parameters detailed above remains rather large reaching up to $10 \%$. These errors are often related to the protocol used (e.g. degree of steadiness of the response during flow ramps, range of 
frequencies and amplitudes during dynamic sweeps), the possibility of wall slip, inertia of geometries.

Thus, exploring new (and preferably simple from a practical perspective) ways of assessing the rheological parameters of an elastoviscoplastic material remains an actual topic.

One simple way of assessing the yield stress of material is the slump test, [12-15]. The applicability of this method is somewhat limited, however, to materials with a large yield stress (typically larger than $100 \mathrm{~Pa}$, such as concrete) when the inertial as well as the surface tension effects may be neglected. Even within this range, the repeatability and accuracy of the yield stress measurements does not exceed roughly $25 \%$, e.g. see Fig. 4 in Ref. [14]. A second simple way of estimating yield stress is to monitor the startup flow of a viscoplastic material on an inclined plane, [16]. Using the lubrication approximation and neglecting both inertial and interfacial effects, the critical angle of the slope corresponding to the flow start up may be related to the yield stress of the material. This method as well is more suited to materials with a higher yield stress. We point out that, to our best knowledge, using the aforementioned tests one can only get an estimate of the yield stress and not of the elastic modulus of the an elasto-viscoplastic material. A third class of simple but insightful experiments that could be exploited to assess the basic rheological parameters of an elasto-viscoplastic fluid relate to studying the dynamics of free fall and impact of Carbopol gel drops on solid surfaces $[17,18]$, on elasto-viscoplastic surfaces [19] or the spreading of elasto-viscoplastic drops on solid surfaces of different wettability [20].

The simplest hydrodynamic experiment able to reveal valuable information on the rheological properties of a fluid relates to monitoring the steady state motion of a spherical object freely falling in the fluid. For a Newtonian fluid and in a range of small Reynolds numbers, this classical hydrodynamic problem has an analytical solution initially obtained by Sir Gabriel G. Stokes in his seminal 1850 paper, [21]. The solution relates the terminal velocity of the object to the viscosity of the fluid providing a very simple method to measure the viscosity of a Newtonian fluid.

A natural question one would like to address is to what extent the Stoke's approach could be used for assessing the basic rheological parameters (yield stress, consistency, power law index and elastic modulus) of an elasto-viscoplastic fluid. Though somewhat counterintuitive, addressing this issue turns out to be a daunting task. The conceptual difficulty arises from the fact that, unlike in the case a spherical object steadily settling within a Newtonian fluid, the flow field is no longer analytically solvable (even for vanishing Reynolds numbers) when either a viscoplastic or an elasto-viscoplastic fluid is considered.

The steady state motion of a spherical object in a Bingham fluid has been solved numerically by Beris and coworkers, [22]. This seminal work revealed a clear departure from the Stokes solution as well as distinct topology of the yield surface. More recently, the dynamics of a drop steadily moving in a viscoplastic fluid has been recently investigated using a lattice Boltzmann approach by Xie and coauthors, [23].

The experimental results presented in $[24,25]$ indicate that the motion of a spherical object in an elasto-viscoplastic fluid (e.g. a Carbopol gel) is even harder to describe theoretically and/or numerically as the presence of the elasticity brings additional features to the flow patterns: a breakdown of the fore-aft symmetry and the emergence of a negative wake qualitatively similar to that observed two decades ago in viscoelastic fluids [26]. This hydrodynamic problem has been solved numerically only recently [27]. An excellent agreement between the simulation results and the experimental ones presented in Refs. [24,25] has been found.

A simpler hydrodynamic setting relates to the impact of a spherical object onto the free surface of an elasto-viscoplastic material. If the initial kinetic energy of the object does not suffice for the establishment of a steady state motion within the gel as in the case of the experiments

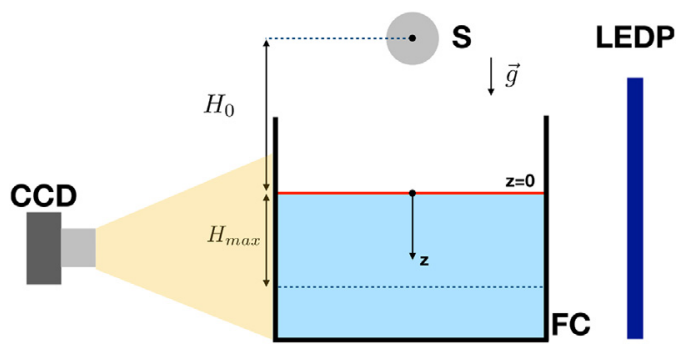

Fig. 1. Schematic illustration of the experimental setup (not in scale): FC - flow container, $\mathbf{S}$ - stainless steel sphere, CCD - digital camera, LEDP - LED panel.

reported in Refs. [24,25], the object will stop at a finite depth within the fluid.

Le Goff and her coworkers have studied the stopping of a spherical object upon its impact onto the free surface of a foam, [28]. Using a simple but yet insightful $1 D$ model that is able to capture the main rheological properties of the foam introduced by Marmottant and Granet [29] they are able fit the experimentally measured trajectories of the spherical object.

The formation of craters upon the fast impact of a spherical object onto the free surface of a Carbopol gel was studied by Tabuteau and coworkers [30].

Here we present a systematic experimental investigation of the stopping process of a spherical object upon its impact onto the free surface of an elasto-viscoplastic material. Furthermore we show how the main rheological parameters of the gel $\left(\sigma_{y}, K, n, G\right)$ can be inferred from the kinematics of the stopping process and introduce for the first time the concept of a "stopping ball rheometer" for an elasto-viscopastic fluid.

The paper is organised as follows. The experimental methods are described in Section 2. The "stopping ball" apparatus, the data acquisition and the data analysis procedures are described in Section 2.1. Measurements of the rheological parameters of the solutions performed by means of classical rotational rheometry are presented in Section 2.2. The rheological characterisation is complemented by the description of a simple elasto-viscoplastic model initially introduced in Ref. [1] able to quantitatively describe the solid-fluid transition. The results of the "stopping ball" experiments are presented in Section 3. Several approaches of inferring the relevant rheological parameters of the elasto-viscoplastic fluid from the analysis of the kinematics of the stopping process are presented. The paper closes with a summary of the main conclusions, Section 4.

\section{Experimental setup and methods}

\subsection{Experimental setup and measurement techinque}

The experimental setup is schematically illustrated in Fig. 1. A stainless steel sphere of radius $R=5.55 \mathrm{~mm}$ and mass $M=5.71 \mathrm{~g}$ (thus the density is $\rho=7973.9 \mathrm{~kg} \cdot \mathrm{m}^{-3}$ ) is released against the free surface of a Carbopol ${ }^{\circledR}$ gel contained in fluid container FC with rectangular cross section $\left(W=20 \mathrm{~cm}, H_{c}=15 \mathrm{~cm}\right)$ and flat transparent walls. The size of the container is significantly larger than the diameter of the spherical object, $W / R \approx 36.36$.

The fluid container is illuminated from the back by a $L E D$ panel LEDP and visualised from the front by a digital camera CCD that records a sequence of images at a speed of $200 \mathrm{fps}$. The total image acquisition time was $2 \mathrm{~s}$. The trajectory of the object is reconstructed by identifying its centre of mass via a standard image processing algorithm written in Matlab. As a post-processing step, each trajectory is interpolated by a smoothing spline function which allows one to reliably compute the first and second order numerical derivatives and ultimately provide the full kinematic picture of the stopping process. 




(a)

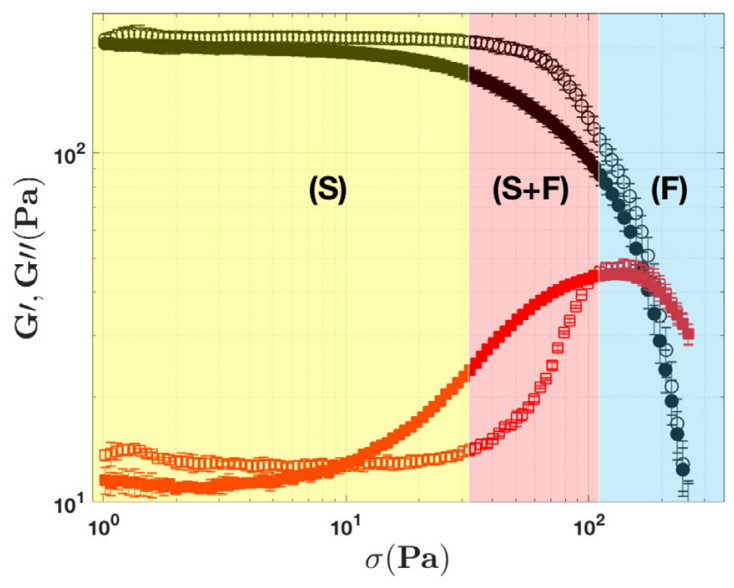

(b)

Fig. 2. (a) Dependence of the absolute value of the rate of shear $|\dot{\gamma}|$ on the applied stress. The full line is a best fit obtained using the elasto-viscoplastic model introduced in Ref. [1]. The dash-dotted line is a best fit obtained using the Herschel-Bulkley model defined by Eq. (1). The red dot marks the point when an elastic recoil is observed on the decreasing branch of the applied stress. (b) Elastic moduli (circles) and viscous moduli (squares) measured during oscillatory stress sweeps. In each panel the empty/full symbols refer to the increasing/decreasing branch of the stress sweep. The measurements were performed with a $0.8 \%$ (wt) Carbopol solution (the solution 3 listed in Table 1). (For interpretation of the references to colour in this figure legend, the reader is referred to the web version of this article.)

\subsection{Choice of the elasto-viscoplastic materials and their rheological characterization}

As working fluids we have used various aqueous solutions of Carbopol ${ }^{\circledR}$ Ultrez10 prepared using the same batch of polymer. To insure the reproducibility of the results, each solution has been prepared according to the same protocol as follows. First, the right amount of anhydrous Carbopol ${ }^{\circledR}$ has been dissolved in deionised water. To enhance the mixing/dissolution process, the solution has been stirred at a speed of $1000 \mathrm{rpm}$ with a commercial propeller mixer. The degree of mixing/dissolution has been assessed visually by monitoring the optical isotropy of the solution. Next, the $\mathrm{pH}$ of the solution has been gradually increased from 3.2 to 7 by titration with small amounts of a 10\% (wt) aqueous $\mathrm{NaOH}$ solution gradually pipetted while gently mixing the solution. The final mixing/neutralisation process never exceeded $1 \mathrm{~h}$. The average density of the Carbopol ${ }^{\circledR}$ solutions was $\rho_{f} \approx 1100 \mathrm{~kg} \mathrm{~m}^{-3}$.

The rheological characterisation of the solutions was performed using a controlled stress rotational rheometer (Mars III, Thermofischer Scientific) controlled stress flow ramps and oscillatory stress sweeps (with a frequency $f=1 \mathrm{~Hz}$ ). To prevent the wall slip, a serrated parallel plate geometry with a gap $d=1 \mathrm{~mm}$ has been used. The absence of any significant wall slip effects during the macroscopic rheological measurements has been proved by checking that the steady state flow curves measured for several distinct gaps perfectly overlap (see the Supplemental Material).

To test the reproducibility and quantitatively assess the instrumental error, each rheological measurement was repeated three times with a fresh sample. Last, to prevent the evaporation of the solvent during the rheological measurements a thin layer of commercial oil has been added to the free meniscus of the sample.

A sample flow curve measured during an increasing/decreasing stress ramp (the averaging time per step stress was $t_{0}=10 \mathrm{~s}$ ) is illustrated in Fig. 2(a). Three distinct deformation regimes can be distinguished. Corresponding to low values of the applied stress, the material behaves as an elastic solid, the region (S) in Fig. 2(a). Corresponding to values of the applied stress significantly larger than the yield stress $\sigma_{y}$ a viscous deformation regime is observed, the region $(F)$. Within this deformation regime, the rheological data can be accurately fitted by the HerschelBulkley correlation, the dash-dotted line in Fig. 2(a). The transition from a solid to a viscous deformation regime is gradual, i.e. it occurs within a band of the applied stresses, the region $(\mathbf{S}+\mathbf{F})$. An additional distinc- tive feature of the solid-fluid transition relates to the lack of reversibility of the deformation states upon increasing/decreasing the applied stresses. The cusp observed on the unloading branch of the flow curve (the red full circle in Fig. 2(a)) relates to an elastic recoil effect. The elasto-viscoplastic nature of the solid-fluid transition together with the weak thixotropy manifested through a hysteresis of deformation states have been observed systematically for various grades of Carbopol and with different rheometers in a sequence of previous publications, $[1,31-$ 37].

To get further insights into the elastic properties of the Carbopol gel, we have performed controlled stress oscillatory sweeps at a frequency $f=1 \mathrm{~Hz}$, Fig. 2(b). Corresponding to the solid deformation regime (S) the elastic modulus $G^{\prime}$ is significantly larger than the viscous modulus $G^{\prime \prime}$ indicating that the material behaves like an elastic solid. Upon an increase of the applied stress past the yield point, the two moduli become comparable in magnitude indicating that the material gradually yields to stress. As for the case of the controlled stress flow ramps, the oscillatory measurements are not fully reproducible upon increasing/decreasing applied stresses which is an intrinsic feature of the solidfluid transition.

To describe the main features of the solid-fluid transition, we resort to a simple $1 D$ model initially introduced in Ref. [1]. The model considers a scalar structural parameter $\bar{\Phi}$ described by an evolution equation

$\frac{d \bar{\Phi}}{d t}=R_{d}+R_{r}+\delta$

Here $R_{d}, R_{r}$ are smooth functions describing the rate of destruction of gel and the rate of reconstruction defined according to:

$$
\begin{aligned}
& R_{d}=-K_{\mathrm{d}}\left[1+\tanh \left(\frac{\sigma / \sigma_{Y}-1}{W}\right)\right] \bar{\Phi} \\
& R_{r}=K_{r}\left[1-\tanh \left(\frac{\sigma / \sigma_{y}-1}{W}\right)\right](1-\bar{\Phi})
\end{aligned}
$$

and $\delta$ is a thermal noise term. The evolution equation for the microstructural parameter $\bar{\Phi}(t)$ is complemented by a Maxwell type thixoelastic constitutive equation inspired from the work of Quemada [38-40]:

$$
\frac{\eta(\dot{\gamma})}{G} \bar{\Phi} \frac{d \sigma}{d t}+\sigma=\eta(\dot{\gamma}) \dot{\gamma}
$$


Table 1

Summary of the Carbopol solutions used in the study and their main rheological parameters obtained via conventional rotational rheometry in conjunction with the model introduced in Ref. [1]. The uncertainties were obtained by repeating each rheological test at least three times.

\begin{tabular}{llllll}
\hline Solution & Concentration $(\% w t)$ & $\sigma_{y}(\mathrm{~Pa})$ & $K\left(\mathrm{Pas}^{n}\right)$ & $n$ & $G^{\prime}(\mathrm{Pa})$ \\
\hline 1 & 0.5 & $48 \pm 5$ & $34 \pm 3$ & $0.37 \pm 0.05$ & $210 \pm 20$ \\
2 & 0.65 & $60 \pm 10$ & $50 \pm 6.5$ & $0.35 \pm 0.06$ & $352 \pm 25$ \\
3 & 0.8 & $100 \pm 18$ & $60 \pm 9$ & $0.38 \pm 0.04$ & $447 \pm 18$ \\
4 & 1 & $200 \pm 15$ & $103 \pm 8.24$ & $0.39 \pm 0.04$ & $530 \pm 30$ \\
5 & 1.5 & $300 \pm 16$ & $124 \pm 7.56$ & $0.35 \pm 0.08$ & $620 \pm 35$ \\
\hline
\end{tabular}

where $G$ is the elastic modulus, $\dot{\gamma}$ the rate of shear and $\eta(\dot{\gamma})=K \dot{\gamma}^{n-1}+$ $\sigma_{y} \frac{1-e^{-m \mid \dot{\gamma}} \mid}{|\dot{\gamma}|}$ is a Papanastasiou regularised Herschel-Bulkley viscosity function.

In order to find the best set of parameters that accurately fit the controlled stress ramps data with the model we have written a nested function program in Matlab. The main function uses the built-in function lsqnonlin in Matlab which solves nonlinear least-squares data fitting problems using a trust-region-reflective algorithm. As input we provide an initial guess for the parameters vector, the target data (see the symbols in Fig. 2(a)) and a function which first solves the Eq. (2) for $\bar{\Phi}$ using the built-in function ode15s in Matlab and then solves Eq. (5) for $\dot{\gamma}$ using the built-in function fzero in Matlab. The output of the main function is a vector with the optimal parameter values. We note that the increasing/decreasing branches of the flow ramp were fitted separately in order to properly capture the hysteresis behavior visible in Fig. 2(a) within the (S) and $(\mathbf{S}+\mathbf{F})$ deformation regimes. The best fitting functions obtained according to this procedure are shown in Fig. 2(a) as full lines. Due to its ability to fit very well the loading/unloading ramps, we will resort to this model later through the paper in order to describe the stopping process of the spherical object.

Through our study we have used five distinct Carbopol solutions. The rheological behavior of each solution can be accurately described by the simple model described above. The yield stress $\sigma_{y}$ ranging in between $49 \mathrm{~Pa}$ and $300 \mathrm{~Pa}$ and the elastic modulus $\mathrm{G}^{\prime}$ ranging in between $210 \mathrm{~Pa}$ and $620 \mathrm{~Pa}$. The relevant rheological parameters of each solution obtained by fitting the flow curves according to the procedure described above together with the uncertainty are given in Table 1 . Note that the concentrations listed in Table 1 are, most probably, smaller than the actual ones as, prior to each rheological test, the solution has been degassed to eliminate air bubbles which lead to a fair amount of evaporation of the solvent. The full description of the rheological measurements performed with all the solutions listed in Table 1 is given in the Supplemental Material.

\subsection{Range of dimensional and non-dimensional parameters}

The rheological characterisation of the elasto-viscoplastic material presented in Section 2.2 allows one to compute the bounds of the relevant non-dimensional parameters governing the impact and stopping of the spherical object.

A systematic discussion on the definition of the non-dimensional numbers governing the flows of viscoplastic materials is presented in Ref. [41].

By varying the release height of the sphere and the Carbopol ${ }^{\circledR}$ concentration, the maximal generalised Reynolds number defined using the speed at impact $U_{0}$ as $R e=\frac{\rho U_{0}^{2}}{K\left(\frac{U_{0}}{R}\right)^{n}}$ varied in the interval $[3.5,13]$. The Bingham number defined as $B n=\frac{\sigma_{y}}{K\left(\frac{U_{0}}{R}\right)^{n}}$ varied in the interval [0.13, 0.4]. The yield number defined as $Y=\frac{3 \sigma_{y}}{2 \operatorname{Rg}\left(\rho-\rho_{f}\right)}$ varied in the interval $[0.21,0.34]$.
The impact phenomenon may be characterised by the inverse Froude number $F r^{-1}=g R / U^{2}$. During our experiments, $F r^{-1}$ was varied in the interval $\mathrm{Fr}^{-1} \in\left[7.9 \cdot 10^{-3}, 7.92 \cdot 10^{-2}\right]$. If one estimates the surface tension $\gamma$ of the gel by $\gamma \approx 0.063 \mathrm{mN}$. $\mathrm{m}$ [42] we find the Weber number

$W b=\frac{2 \rho_{f} R U^{2}}{\gamma}$

in the range $W b \in\left[1.33 \cdot 10^{2}, 1.33 \cdot 10^{3}\right]$ which indicates that the effects of the surface tension are negligible during our experiments.

\section{Results}

\subsection{Kinematics of the stopping process}

A sequence of images of the spherical object acquired prior and after its impact on the free surface of the gel is illustrated in Fig. 3. Upon its impact at $t=0$ (panels (b-c)) the spherical object generates a crater into the surface of the Carbopol ${ }^{\circledR}$ gel. This observation is consistent with the previous experimental observations by Tabuteau and coworkers, [30]. At later stages of the motion, an air drop is entrapped into the gel (panel d) and the spherical object oscillates around a final resting position (panel (e)). As explained in Section 2.1, the subsequent images of the spherical object allow one to determine the trajectories of the object and, consequently, its speed and acceleration. Thus, we obtain the full kinematic picture of the stopping process.

The kinematic of the stopping process of the object upon impacting the free surface of the gel is illustrated in Fig. 4. As the initial kinetic energy of the particle is insufficient for the establishment of a constant speed settling regime as was the case of the experiments reported in Ref. $[1,25]$, the particle bounces with a period $T$ and finally stops at a finite depth $H_{0}$, panel (a). The measurement of the particle trajectory allows one to compute the speed by numerical differentiation, $U=\frac{d z}{d t}$ and consequently to estimate a scale for the shear rate as $\dot{\gamma} \approx \frac{U}{R}$, [28]. To support the choice of the scale for the shear rate, Le Goff and coworkers resorted to an ingenious method of visualising the deformation field by seeding the foam with dark particles, Fig. 7 in Ref. [28]. Although we did not perform such visualisation experiments, we believe the assumption that the yielded region is comparable in size to the diameter of the sphere is reasonable in our case too. This is supported by the systematic measurements of the flow fields around a sphere moving at a constant speed in a Carbopol gel performed by Putz et al. [24] (see Fig. 8 therein).

Upon the impact, the average shear rate first decays rapidly from a rather large value $\dot{\gamma}=178.5 \mathrm{~s}^{-1}$ to zero and then oscillates around its final stopping value $\dot{\gamma}=0 \mathrm{~s}^{-1}$. The early stage of the motion characterised by a shear rate significantly larger than unity $\dot{\gamma}>1 \mathrm{~s}^{-1}$ corresponds to a fully yielded or viscous deformation regime (VR) described by the Herschel constitutive relationship (the highlighted region (F) in Fig. 2(a)). During the final stages of the motion, $\dot{\gamma}<1 \mathrm{~s}^{-1}$ which corresponds to an unyielded or elastic solid regime (ER) characterised by Hooke's law (the highlighted region (S) in Fig. 2(a)).

The acceleration during the stopping process is computed by numerical differentiation of the speed, $a=\frac{d U}{d t}$. 
(a)

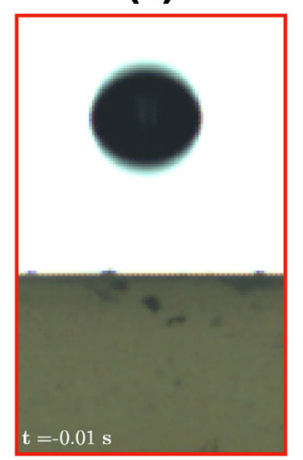

(b)

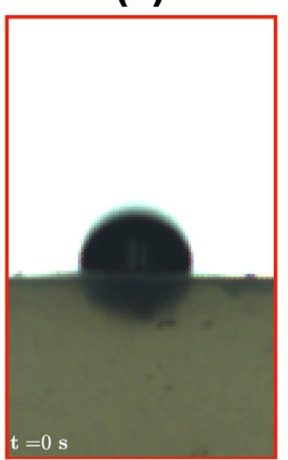

(c)

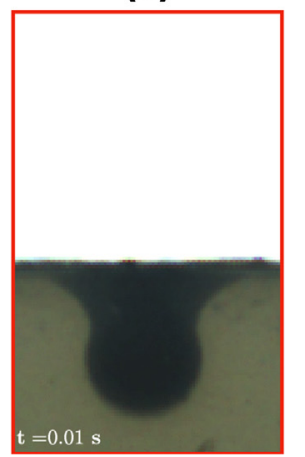

(d)

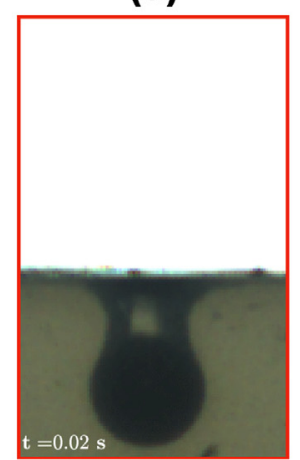

(e)

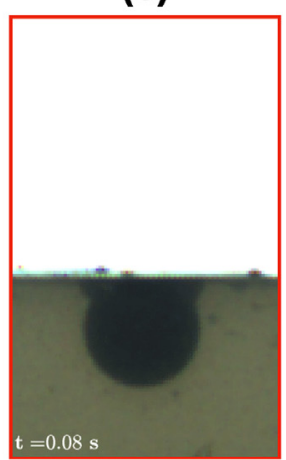

Fig. 3. Sequence of images of the spherical object. The time instants are indicated in the inserts. The time of impact is $t_{0}=0 \mathrm{~s}$.

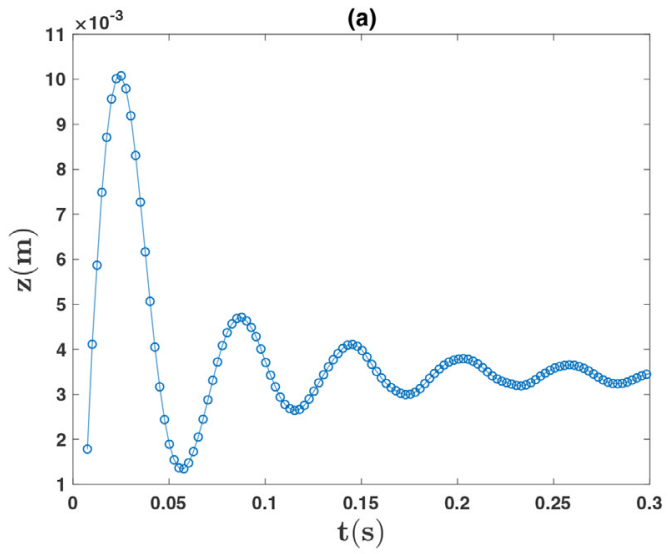

(b)



(c)

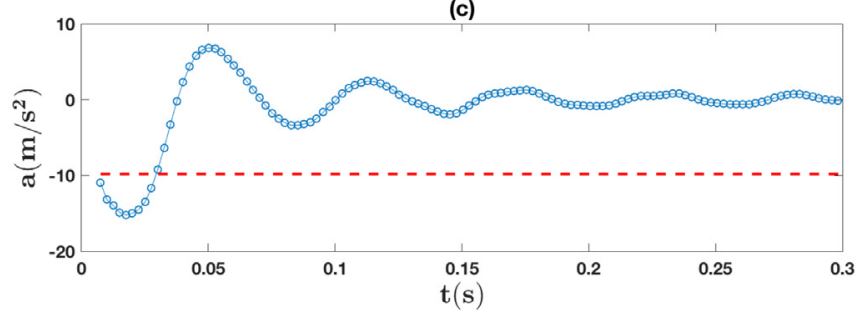

Fig. 4. (a) Trajectory of the particle after its impact onto the gel surface. (b) Temporal evolution of the rate of shear: (VR) - viscous regime, (ER) - elastic regime. (c) Temporal evolution of the particle acceleration during the stopping process. The dashed line points to the acceleration of the gravity.

The computed speeds and accelerations allow one to associate to each experimental test performed with various Carbopol solutions for different release heights $H_{0}$ a phase portrait $(U, a)$.

\subsection{Description of the stopping process in the framework of a simple elasto-viscoplastic model, [1]}

The equation of motion of the sphere during the stopping process within the Carbopol gel may be written:

$M \frac{d U}{d t}=F+F_{b}$ where $F$ is the total force acting on the sphere and $F_{b}=\left(M-M_{\text {water }}\right) g=$ $4 \pi R^{3} / 3\left(\rho-\rho_{\text {water }}\right) g$ is the buoyancy corrected gravity force. Following Goff et al. [28], the total force acting on the sphere may be written in terms of the characteristic scales of the stresses exerted by the sphere as: $F=-\alpha \pi R^{2} \sigma$ with $\alpha$ a geometric parameter characterising the effective contact area between the sphere and the gel and $\sigma$ the stress exerted on the moving sphere. Unlike for the case of the low Re settling of a spherical object in a Newtonian fluid, this parameter is not known $a$ priori and will be obtained by fitting the experimental data.

The equation of motion reduces to:

$\frac{d U}{d t}=-\frac{\alpha \pi R^{2} \sigma}{M}+\left(1-\frac{\rho_{\text {water }}}{\rho}\right) g$

As discussed in Section 3.1, the scale for the rate of shear may be estimated as $\dot{\gamma}=U / R$.

As a constitutive framework we use the simple elasto-viscoplastic model initially proposed by Putz and Burghelea in Ref. [1] and briefly introduced in order to describe the rheological measurements in Section 2.2.

According to Gonzalez et al. [32] this simple scalar model may be written in a tensorial form as:

$\bar{\Phi} \nabla=-\frac{G}{\eta(\dot{\varepsilon})} \sigma-G \dot{\varepsilon}$

where $\bar{\Phi}$ is the average volume fraction of the un-yielded gel around the sphere, $G$ is the elastic modulus of the gel, $\dot{\varepsilon}$ is the rate of strain tensor and $\boldsymbol{\sigma}$ is the upper-convected derivative of the stress tensor:

$\underset{\sigma}{\nabla}=\frac{D \sigma}{D t}-(\nabla U)^{T} \cdot \sigma-\sigma \cdot(\nabla U)$

Here $D \cdot / D t$ is the material derivative:

$\frac{D \sigma}{D t}=\frac{\partial \sigma}{\partial t}+U \cdot \nabla \sigma$

The contribution of the convective terms $-(\nabla U)^{T} \cdot \sigma-\sigma \cdot(\nabla U)$ may be crudely approximated by $-2 \sigma \dot{\gamma}$. During an infinitesimal time $\delta t$, a volume of fluid $|\boldsymbol{U}| \delta t \times \pi(d+R)^{2}$ enters the yielded zone $\Omega$ while the same amount exits. Here $d$ stands for the characteristic size of the yielded region whose scale is set by the radius of the sphere, $d \approx R$. After integration over the yielded zone, the convective term $\boldsymbol{U} \cdot \boldsymbol{\nabla} \boldsymbol{\sigma}$ may be approximated by:

$\boldsymbol{U} \cdot \boldsymbol{\nabla} \sigma \approx \frac{|U| \pi(d+R)^{2}}{V_{d}} \sigma$

where $V_{d}$ is the volume of the yielded zone.

Finally by plugging Eq. (9) in Eq. (12) one obtains:

$\frac{d \sigma}{d t} \approx \frac{1}{\bar{\phi}}\left[-\frac{G}{\eta(\bar{\Phi}, \dot{\gamma})} \sigma-G \dot{\gamma}\right]-\frac{3|U|}{4(d+R)} \sigma-2 \dot{\gamma} \sigma$ 
In Eq. (13), the viscosity $\eta(\bar{\Phi}, \dot{\gamma})$ depends on the characteristic strain rate in the fluid phase. Corresponding to a fluid state, we define the strain rate $\dot{\gamma}_{\text {vis }}$ :

$\dot{\gamma}_{\text {vis }}=(1-\bar{\Phi}) \times \dot{\gamma}$

such that when fully solid $(\bar{\Phi}=1)$, there exists no viscous strain rate and in a fully liquid state the viscous strain rate equals the total strain rate. Thus the viscosity $\eta$ can now be expressed using, for instance, a regularized visco-plastic formulation [43]

$\eta(\bar{\Phi}, \dot{\gamma})=\eta\left(\dot{\gamma}_{v i s}\right)=\sigma_{y} \frac{1-e^{-m \dot{\gamma}_{v i s}}}{\dot{\gamma}_{v i s}+\epsilon}+K\left(\dot{\gamma}_{v i s}+\epsilon\right)^{n-1}$

where $\epsilon \ll 1$ is a stabilization parameter and $m$ is the regularisation parameter. When $m \rightarrow+\infty$, this law approximates the Herschel-Bulkley fluid behavior.

Following Putz and Burghelea [1], the evolution of volume fraction of the solid phase $\bar{\Phi}$ can be modelled by:

$\frac{D \bar{\Phi}}{D t}=-\underbrace{R_{d}}_{\text {destruction }}+\underbrace{R_{r}}_{\text {reconstruction }}+\underbrace{\delta}_{\text {stabilization }}$.

Finally, integrating the destruction and reconstruction terms $R_{d}, R_{r}$ given by Gonzalez et al. [32], the evolution equation for the average solid fraction $\bar{\Phi}$ may be written:

$$
\begin{aligned}
\frac{d \bar{\Phi}}{d t}= & -K_{\mathrm{d}}\left[1+\tanh \left(\frac{|\sigma| / \sigma_{Y}-1}{W}\right)\right] \bar{\Phi} \\
& +K_{r}\left[1-\tanh \left(\frac{|\sigma| / \sigma_{y}-1}{W}\right)\right](1-\bar{\Phi}) \\
& +\delta \\
& +\frac{3|U|}{4\left(d+R_{0}\right)}(1-\bar{\Phi})
\end{aligned}
$$

In order to solve the coupled problem given by Eqs. (9), (16) and (8), the unknown vector $\boldsymbol{X}$ is defined as the concatenation

$$
\boldsymbol{X}=\left\{\begin{array}{c}
\bar{\Phi} \\
\sigma \\
V \\
z
\end{array}\right\} .
$$

The evolution of $\boldsymbol{X}$ is described by the system of first order non-linear ordinary differential equations:

$\frac{d \boldsymbol{X}}{d t}=\left\{\begin{array}{c}d \bar{\Phi} / d t \\ d \sigma / d t \\ d U / d t \\ U\end{array}\right\}$

where the first three columns are given by Eqs. (9), (16) and (8). Considering $t=0$ the time of impact, initial conditions are as follow.

1. The material, initially at rest is fully solid:

$$
\bar{\Phi}(t=0)=1 \text {. }
$$

2. The elasto viscoplastic material is initially relaxed:

$$
\sigma(t=0)=0
$$

3. The velocity at impact is set by the release height $H_{0}$

$$
\left.U\right|_{t=0}=-\sqrt{2 g H_{0}}
$$

4. The initial position is

$$
\left.z\right|_{t=0}=0
$$

The initial conditions thus write

$$
\left.\boldsymbol{X}\right|_{t=0}=\left\{\begin{array}{c}
1 \\
0 \\
-\sqrt{2 g H_{0}} \\
0
\end{array}\right\}
$$

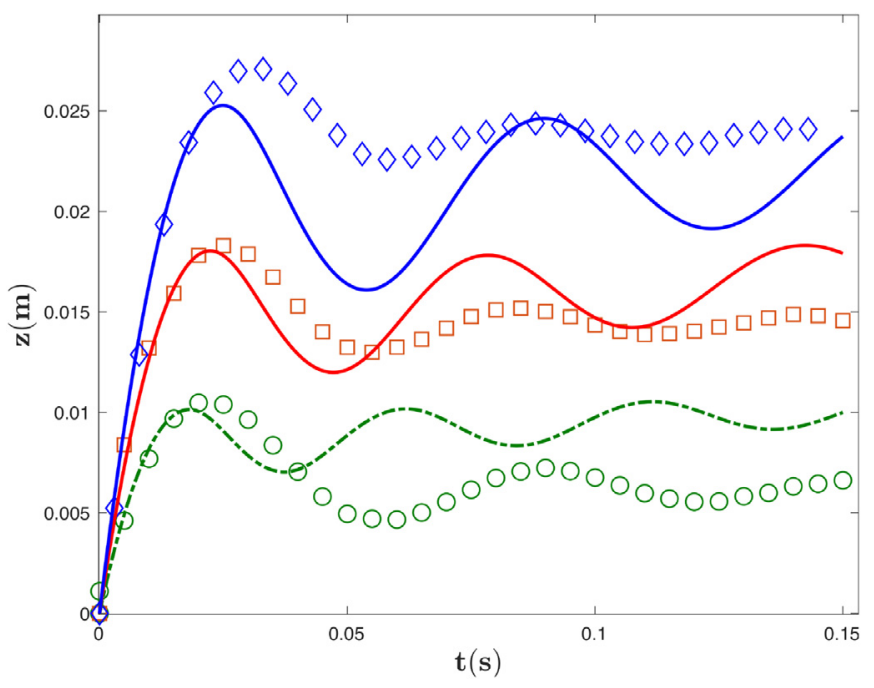

Fig. 5. Comparison between the experimental measurements (symbols) using the solution 4 (see Table 1) and the predictions of the model (full lines). The symbols refer to different release heights: circles $-H_{0}=6.5 \mathrm{~cm}$, squares $-H_{0}=$ $13.5 \mathrm{~cm}$, diamonds $-H_{0}=21 \mathrm{~cm}$.

After turning it dimensionless using characteristic magnitudes of the problem, Eq. (18) is solved using an explicit fourth order Runge-Kutta numerical method in Matlab. As the spatial field is not taken into account, the problem depends only on time and has four scalar unknown. The computation is very fast (less than a second) and enables quick testing of various parameters.

In addition, the direct problem presented above can provide a characterisation tool for material or physical properties. An inverse method consisting of an optimisation loop is implemented. The error is defined as the norm two of the residuals between the experimentally measured depth versus time and the simulation result. This error is minimised via a mean square regression algorithm (the built-in gradientless NelderMead Simplex Method in Matlab).

In Fig. 5 we compare the predictions of the model with the experimental measurements. The data was acquired using the solution 4 (see Table 1). The early stages of the motion are rather well described by the model. The periodic motion observed in the late stages of the motion is recovered, though a phase shift with respect to the data is systematically observed.

A possible reason for this discrepancy may be understood as follows. We have reduced the details of the complex flow field to a limited number of coefficients in a lumped parameters model. In the simplified derivation of Eq. (13) we did not account for the topology of the flow field around the stopping object and artificially introduced a space scale associated to the "flowing" region, $d \approx R$. The experimental measurements of the flow field around an object moving through a Carbopol gel reported in Ref. [24] reveal, however, a rather complex flow topology. A distinctive feature of the flow pattern relates to the negative wake observed behind the moving object which is associated to the generation of extensional stresses. The simplified derivation presented above did not account for this effect which, during the intermediate stages of the stopping process, might bring a significant contribution to the total stress. In the absence of an analytical solution for the flow field, this deficiency of the approach can not be corrected and the agreement between experiments and numerical simulations will always remain partial.

\subsection{A simplified assessment of the rheological properties of the material}

Though reasonably reliable, the approach described in Section 3.2 remains rather difficult to implement as it requires solving iteratively a strongly nonlinear coupled optimisation problem. We propose in the fol- 


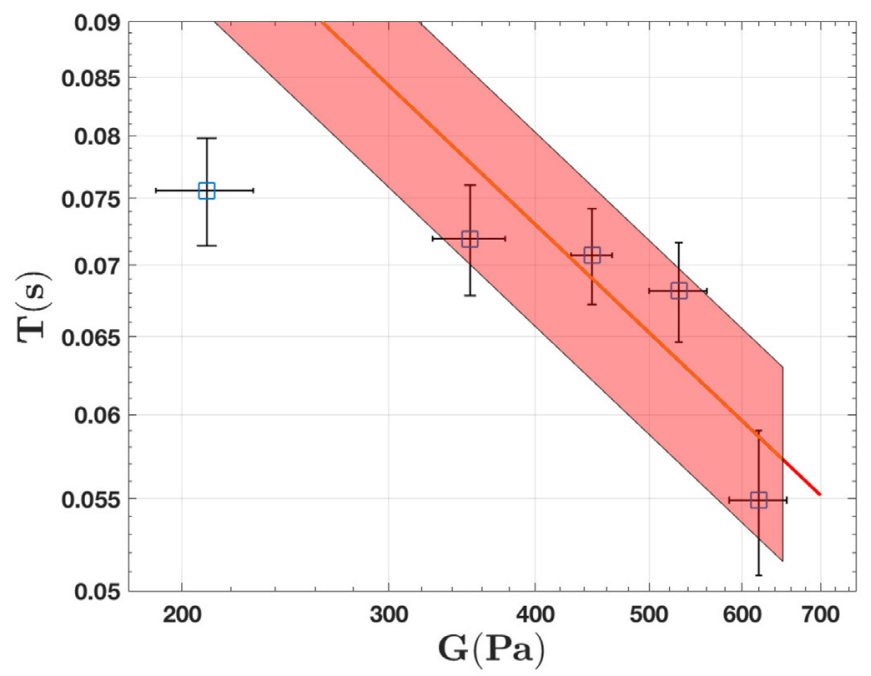

Fig. 6. Dependence of the period of oscillations measured from the stopping ball experiments on the elastic modulus measured via small amplitude oscillatory tests. The full line is the fit according to the theoretical prediction, Eq. (25). The shaded region highlights the error bounds of the nonlinear fit computed using the error of the fit parameters. The horizontal error bars are defined by the uncertainties of the classical rheological measurements of the yield stress and consistency (see Table 1).

lowing a simpler method of inferring the main rheological parameters of the solution from measurements of the trajectories of the stopping object. The main idea behind is to notice that the motion of the object may be decoupled in two parts. As already emphasised in Section 3.1 and illustrated in Fig. 4(b), at early stages of the stopping process the fluid deformation is predominantly viscous whereas at the latest stages it may be considered purely elastic. Through the rest of this section, we will study each regime separately, derive analytical solutions for the equation of motion and provide a strategy for assessing the main rheological parameters of the material from measurements of the kinematics of the stopping process.

\subsubsection{The elastic regime}

Within the asymptotic limit $\bar{\Phi} \rightarrow 1$ that corresponds to the elastic deformation regime the constitutive equation reduces to Hooke's law and the stress acting on the sphere becomes $\sigma=\alpha G \gamma$ where $G$ is the elastic modulus and $\gamma$ is the strain. Consequently, by neglecting the viscous damping, the equation of motion of the spherical object reduces to:

$\frac{d^{2} U}{d t^{2}}=-\frac{\pi \alpha R G}{M} U$

The equation above has been obtained by differentiating the second law of dynamics given by Eq. (8) with time and bearing in mind that buoyancy term is time independent. Eq. (24) is the equation of a harmonic oscillator with the period of oscillations given by:

$T=2 \pi R \sqrt{\frac{4 \rho}{3 \alpha}} G^{-1 / 2}$

The elastic modulus $G$ of each gel has been measured via conventional small amplitude oscillatory measurements, see Fig. 2(b). We note that for each gel, the measured period of oscillations did not depend on the initial release height $H_{0}$ of the object which corroborates with the assumption that the early viscous stage and late elastic stage of motion may be indeed de-coupled. Measurements of period of oscillation of the position of spherical object around its equilibrium value $H_{\max }$ performed for several gels are presented in Fig. 6 . The full line is the best fit to the theoretical prediction given by Eq. (25) performed using the built in Matlab fitnlm routine together with the Levenberg-Marquardt algorithm. We note that the measured period of oscillation is fairly well

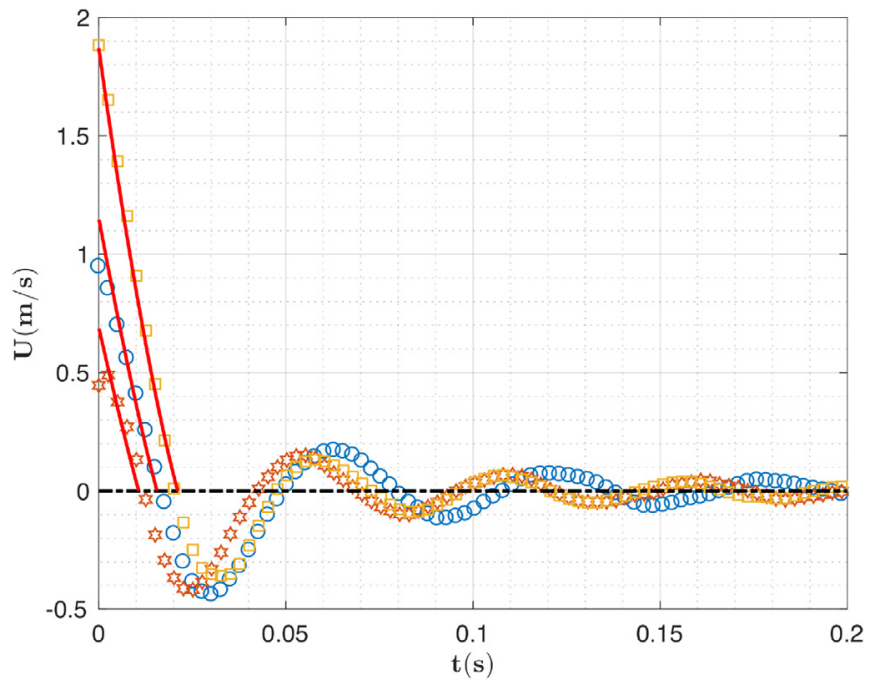

Fig. 7. Instantaneous velocity of the spherical object measured from the moment of its impact with the surface of a Carbopol gel $\left(\sigma_{y}=300 \mathrm{~Pa}\right)$ for three distinct heights of release: squares - $H_{0}=28.5 \mathrm{~cm}$, circles $-H_{0}=16.5 \mathrm{~cm}$, stars $H_{0}=11 \mathrm{~cm}$. The full lines are implicit hypergeopmetric fit functions according to Eq. (29).

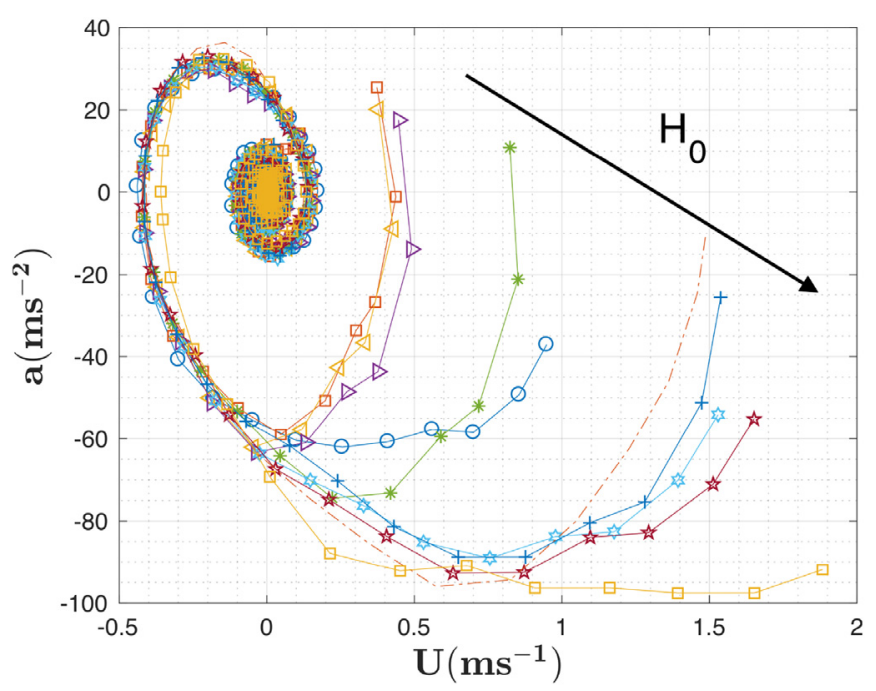

Fig. 8. Phase portrait of the stopping process in a solution with $\sigma_{y}=$ $300 \mathrm{~Pa}( \pm 16 \mathrm{~Pa})$. The symbols refer to the release height $H_{0}$ : orange squares - $H_{0}=11 \mathrm{~cm}$, yellow left triangles - $H_{0}=13.5 \mathrm{~cm}$, magenta right triangles $H_{0}=15 \mathrm{~cm}$, green stars $-H_{0}=16.5 \mathrm{~cm}$, blue circles $-H_{0}=18 \mathrm{~cm}$, orange dots - $H_{0}=19.5 \mathrm{~cm}$, blue pluses $-H_{0}=21 \mathrm{~cm}$, light blue stars $-H_{0}=24 \mathrm{~cm}$, dark red stars $-H_{0}=25.5 \mathrm{~cm}$, yellow squares $-H_{0}=27 \mathrm{~cm}$. (For interpretation of the references to colour in this figure legend, the reader is referred to the web version of this article.)

fitted by the theoretical prediction except for the smallest value of the elastic modulus (the smallest yield stress).

The deviation of this value from the theoretical prediction may be explained as follows. The yield stress of this Carbopol gel was too small and unable to fully stop the spherical object even for the smallest value of the release height. Thus, a small viscous drift always existed in the case, making a proper decoupling of the viscous and elastic stages of motion impossible. The slow viscous drift effect was not observed for the rest of the gels we have tested.

The curve defined by Eq. (25) may be used as a first calibration curve of the "stopping ball" rheometer. Once the value of the parameter $\alpha$ is obtained by fitting a set of data with known elastic moduli, the elastic 
modulus of any new solution may be obtained by measuring the period of oscillations of the stopping object. This simple procedure provides estimates of the elastic modulus with an accuracy of roughly $20 \%$ provided that spherical object reaches a full stop (no viscous drift is observed).

\subsubsection{The viscous regime}

During the early stages of the motion when $\dot{\gamma}>1 \mathrm{~s}^{-1}$ the response of the material is dominantly viscous $(\bar{\Phi} \rightarrow 0)$ and the stress is related to the rate of deformation via the Herschel-Bulkley constitutive law, $\sigma=\sigma_{y}+K\left(\frac{|U|}{R}\right)^{n}$. Consequently, the equation of motion reduces to:

$\dot{U}+A|U|^{n}=B$

with

$A=\frac{\alpha \pi}{R^{n-2}} \frac{K}{M}=\frac{3 \alpha K}{4 R^{n+1} \rho}$

$B=\left(1-\frac{\rho}{\rho_{f}}\right) g-\frac{\pi R^{2} \alpha}{M} \sigma_{y}=\left(1-\frac{\rho}{\rho_{f}}\right) g-\frac{3 \alpha}{4 \rho R} \sigma_{y}$

The general solution of Eq. (26) is:

$\left.{ }_{2} F_{1}\left[1, \frac{1}{n}, 1+\frac{1}{n} ; \frac{A}{B} U^{n}\right] U\right|_{U_{0}} ^{U}=B\left(t-t_{0}\right)$

where $U_{0}=\sqrt{2 g H_{0}}$ is the initial speed of the sphere at the moment of impact $\left(t_{0}=0\right)$ and ${ }_{2} F_{1}$ is the Euler hypergeometric function defined by:

${ }_{2} F_{1}(a, b ; c ; z)=\sum_{k=0}^{\infty} \frac{(a)_{k}(b)_{k}}{(c)_{k}} \frac{z^{k}}{k !}$

where $(x)_{k}=\frac{\Gamma(x+k)}{\Gamma(x)}$ stands for the Pochammer symbol.

Several times series of the velocity of the spherical object measured after its impact onto a Carbopol gel with $\sigma_{y}=300 \mathrm{~Pa}$ together with the nonlinear fitting functions using the implicit analytical solution Eq. (29) are illustrated in Fig. 7. We note that corresponding to each release height $H_{0}$ explored in Fig. 7, the analytical solution fits very well the experimental data. Note that, as the hypergeometric Euler function becomes complex valued for negative arguments, only the positive parts of the velocity are fitted. The yield stress obtained from the three fitting functions shown in Fig. 7 is $\sigma_{y}=270 \pm 20 \mathrm{~Pa}$ which is reasonably close to the value obtained via conventional rheometry. The consistency obtained from the three fits is $K=150 \pm 30 \mathrm{Pas}^{n}$ and the power law index is $n=0.5 \pm 0.2$. We note that the values obtained for the power law index and the consistency are less accurate, within 30\% different from the ones obtained via conventional rheometry.

The computed speeds and accelerations of the stopping object allow one to build a phase portrait of the stopping process. This phase portrait represents a unique kinematic fingerprint of the stopping process and it encodes the main rheological parameters of the gel: yield stress, consistency, power law index and elastic modulus. Such a phase portrait is presented in Fig. 8 for a Carbopol gel with $\sigma_{y}=300 \mathrm{~Pa}( \pm 10 \mathrm{~Pa})$.

According to Eq. (26), the acceleration at the moment when the speed $U=0 \mathrm{~m} / \mathrm{s}$ is given by: $a_{0}=\left.a\right|_{U=0}=B$ which is independent on the release height $H_{0}$. This is qualitatively visible in Fig. 8: all phase portraits cross the vertical axis at the same $a_{0} \approx-60 \mathrm{~m} / \mathrm{s}^{2}$. Quantitatively, one obtains $\sigma_{y}=330 \mathrm{~Pa}$ whereas we have measured $\sigma_{y}=300 \mathrm{~Pa}$ via conventional rheometry. This very simple approach based on plotting the phase portrait is only able to give a very crude estimate for the yield stress but is unable to provide reliable values for the consistency and the power law index.

Yet an even simpler way to obtain a crude estimate of yield stress the from the analysis of the stopping ball trajectory comes from writing the energy conservation equation:

$M g H_{0}+\left(M-M_{f}\right) g H_{\max }=\alpha \pi R^{2} \sigma_{y} H_{\max }+\alpha \pi R^{2-n} K \int_{0}^{H_{\max }}|U|^{n} d z$

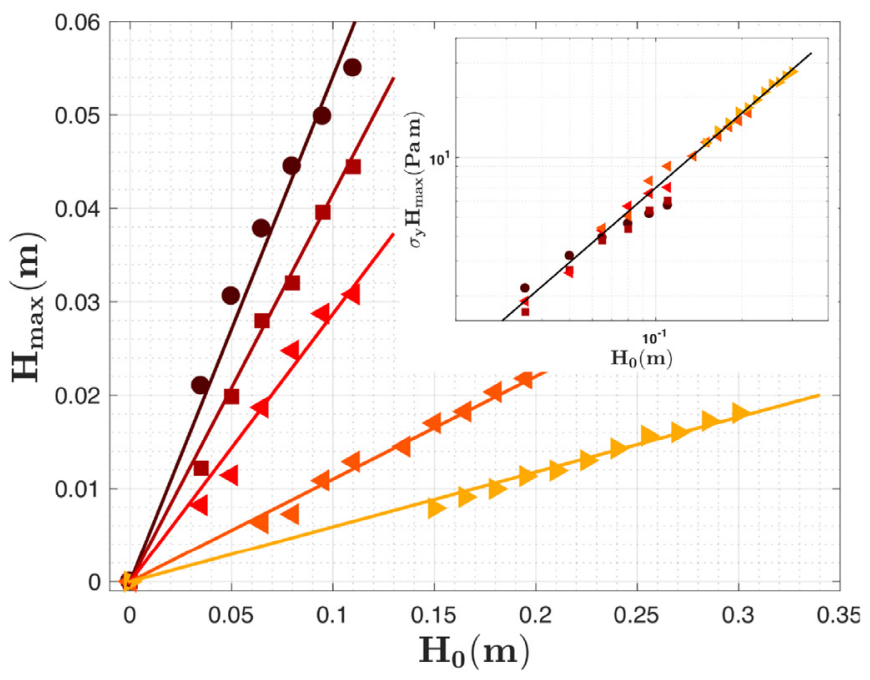

Fig. 9. Dependence of the maximum penetration depth $H_{\max }$ on the initial release height $H_{0}$ measured for several values of the yield stress: The full lines are linear fitting functions $H_{\max }=S H_{0}$. The insert presents the same data rescaled by the yield stress $\sigma_{y}$.

Note that in the equation above we have neglected the elastic dissipative term. The integral term in the right hand side of Eq. (31) is rather difficult to compute using the implicit solution for the sphere velocity given by Eq. (29). A reasonable approximation would be to assume instead a classical viscous damping solution for the speed in the form $U=U_{0} e^{-\frac{t}{\tau}}$ where $\tau$ is a damping time scale related to the viscous damping. The characteristic damping time may be estimated as $\tau \approx \frac{2 \rho_{f} R^{2}}{9 \eta_{e f f}}$ where the scale of the effective viscosity $\eta_{\text {eff }}$ is set by the maximal rate of shear at the impact, $\eta_{\text {eff }} \approx K\left(\frac{U_{0}}{R}\right)^{n-1}$.

With these assumption it is straightforward to derive the relationship between the maximum penetration depth $H_{\max }$ and the initial release height $H_{0}$ :

$H_{\text {max }} \approx \frac{M g H_{0}-\alpha C \sqrt{H_{0}}}{\alpha \pi R^{2} \sigma_{y}-\left(M-M_{f}\right) g}$

where $C=\frac{\pi R^{4}}{9 n} \sqrt{8 g} \approx 10^{-6} \mathrm{Jm}^{-2}$. Thus, to a leading order in $\sqrt{H_{0}}$, the maximal penetration depth may be written:

$H_{\text {max }} \approx \frac{M g H_{0}}{\alpha \pi R^{2} \sigma_{y}-\left(M-M_{f}\right) g}$

To test this proportionality relation, we plot in Fig. 9 the dependence of the maximum penetration depth $H_{\max }$ on the release height $H_{0}$ measured for various values of the yield stress.

Regardless the yield stress of the Carbopol ${ }^{\circledR}$ gel, the dependencies observed in Fig. 9 are linear and their slopes $S$ decrease monotonically with the yield stress $\sigma_{y}$ which is a first validation of the approximation expressed by Eq. (33). The apparent power law scaling $S \propto \frac{M g}{\alpha \pi R^{2}} \sigma_{y}^{-1}$ of the slopes of the linear dependencies with the yield stress suggested by the Eq. (33) is confirmed by plotting $\sigma_{y} H_{\max }$ versus $H_{0}$, the inset in Fig. 9. When rescaled by $\sigma_{y}$ all data collapse onto an universal curve.

The dependence of the linear slope $S$ on the yield stress is illustrated in Fig. 10(a). The full line is a power law fit $S=Q \sigma_{y}^{-m}$ with $Q=$ $38.3 \pm 5.3(\mathrm{~Pa}), m=1.1 \pm 0.3$. The power law fit has been computed using the built in Matlab function fitnlm in conjunction with the LevenbergMarquardt algorithm. The error bounds computed using the errors of the nonlinear fit parameters are highlighted by shaded area. This dependence may be understood as a second calibration curve of the "stopping ball rheometer" for an elasto-viscoplastic fluid and provides a simple way of assessing the yield stress solely from measurements of the maximal penetration depths performed for several release heights $H_{0}$. 




(a)

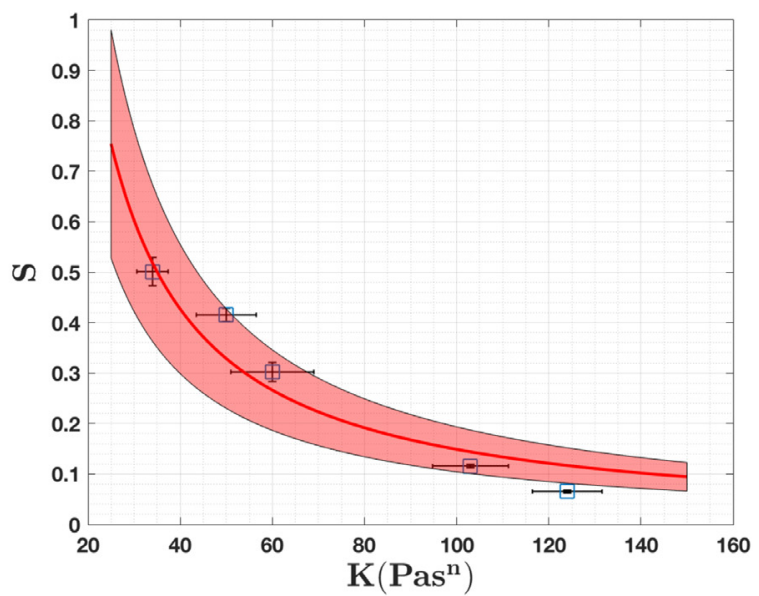

(b)

Fig. 10. (a) Dependence of the slope $S$ of the linear dependence of $H_{\max }$ on the initial release height $H_{0}$ on the yield stress of the gel $\sigma_{y}$. The full line is a power law fit, $S \propto \frac{M g}{2 \alpha \pi R^{2}} \sigma_{y}^{-1}$. (b) Dependence of the slope $S$ of the linear dependence of $H_{\max }$ on the initial release height $H_{0}$ on the consistency of the gel $K$. The full line is a power law fit, $S \propto 23 \mathrm{~K}^{-1.2}$. In both panels the shaded regions highlight the error bounds of the nonlinear fit and the vertical error bars are defined via the error of the linear fitting of the data presented in the main plot of Fig. 9. The horizontal error bars are defined by the uncertainties of the rheological measurements (see Table 1).

The dependence of the slope $S$ on the consistency of the Carbopol ${ }^{\circledR}$ gel is illustrated in Fig. 10(b). Unlike for the case of the dependence $S=S\left(\sigma_{y}\right)$ illustrated in Fig. 10(a) we could not derive an analytical scaling for the dependence $S=S(K)$. However, we noted that the data presented in Fig. 10(b) may be fitted by a power law as well, though the confidence interval (the highlighted region) is significantly broader.

\section{Conclusions, outlook}

A simple method of simultaneously assessing all main rheological parameters $\left(\sigma_{y}, K, n, G^{\prime}\right)$ of an elasto-viscoplastic was presented.

Whereas a spherical object steadily moving at low Re through a elasto-viscoplastic material is a rather difficult to use as one needs to numerically solve the full hydrodynamic problem coupled to an appropriate elasto-viscoplastic model, monitoring the stopping process of a spherical object provides a number of relatively simple ways of assessing the main rheological parameters of the gel. From a technical perspective, the idea is rather simple as it only requires imaging the stopping object (in most cases an imaging speed of 200 fps does suffice, meaning that a regular smartphone may be used), measuring the time series of its position and computing the time series of speeds and accelerations.

By coupling the equation of motion of the spherical object to the micro-structural model initially proposed by Putz and Burghelea, [1,32], we derive an approximate form for the equation of motion and solve it numerically. The optimal numerical solution obtained via a nonlinear optimisation algorithm is in a fair agreement, though discrepancies are systematically visible, Fig. 5 . We believe that these discrepancies originate in the nontrivial features of the flow pattern (such as the emergence of a negative associated with elasticity of the gel and the extensional stresses) not accounted for in the simplified approach presented in Section 3.2.

A further simplification of the problem comes from noticing that the early stages of the motion are dominated by viscous effects whereas the late stages by purely elastic ones. The two types of behaviors can thus be fully de-coupled and one may study the asymptotic limits $\bar{\Phi} \rightarrow 1$ and $\bar{\Phi} \rightarrow 0$ separately. Corresponding to each asymptotic limit the equation of motion is analytically solvable allowing a direct comparison with the experimentally observed flow kinematics.

In the first asymptotic limit, the equation of motion reduces to that of an harmonic oscillator with the period related to the elastic modulus of the gel via Eq. (25). This result provides a quick, cheap and technically simple method of estimating the elastic modulus of a gel by measuring the period of oscillations of the stopping object around its equilibrium position, Fig. 6.

In the second asymptotic limit the equation of motion has an analytical solution given implicitly by Eq. (29) which fits very well the viscous part of the velocity time series, Fig. 7. The yield stress obtained from the fit procedure is close (within $3.5 \%$ ) to the value obtained via conventional rheometry. A simpler way of obtaining an estimate for the yield stress that requires no sophisticated fitting by an implicit equation that involves a special function is to plot the phase portraits $(U, a)$ corresponding to different release heights, Fig. 8. The intersection of each phase portrait with the vertical axis $U=0$ provides a reliable estimate for the yield stress via Eq. (28).

The maximum penetration depth $H_{\max }$ of the object is found to depend linearly (to a leading order in $\sqrt{H_{0}}$ ) on the release height $H_{0}$, Eq. (33). This theoretical result is rather well supported by the experimental measurements, Fig. 9. This allows one to build a calibration curve for the "stopping ball rheometer" by plotting the value of the slope $S$ versus the yield stress, Fig. 10(a).

In closing we note that although the accuracy of this method cannot compete with that of the classical rheological tests, its simplicity might appeal in cases estimates for the main rheological parameters of an elasto-viscoplastic material do suffice.

\section{Acknowledgements}

We are indebted to Dr. Cathy Castelain for numerous discussions and valuable feedback. We are particularly indebted to the second reviewer for her/his constructive remarks that led to a significant improvement of our paper. One of us (T.B.) is indebted to Dr. Volfango Bertola for a number of enlightening discussions and a critical assessment of our results.

\section{References}

[1] A.M.V. Putz, T.I. Burghelea, The solid-fluid transition in a yield stress shear thinning physical gel, Rheol. Acta 48 (2009) 673-689. 
[2] Q.D. Nguyen, D.V. Boger, Measuring the flow properties of yield stress fluids, Annu. Rev. Fluid Mech. 24 (1992) 47-88.

[3] P. Coussot, Yield stress fluid flows: a review of experimental data, J. Non Newton Fluid Mech. 211 (2014) 31-49.

[4] N.J. Balmforth, I.A. Frigaard, G. Ovarlez, Yielding to stress: recent developments in viscoplastic fluid mechanics, Annu. Rev. Fluid Mech. 46 (2014) 121-146.

[5] D. Bonn, J. Paredes, M.M. Denn, L. Berthier, T. Divoux, S. Manneville, Yield stress materials in soft condensed matter, [cond-mat.soft] arXiv:1502.05281(2015).

[6] H.A. Barnes, The yield stress-a review or ' $\pi \alpha v \tau \alpha \rho \epsilon l$ '-everything flows? J. Non Newton Fluid Mech. 81 (1999) 133-178.

[7] H.A. Barnes, K. Walters, The yield stress myth? Rheol. Acta 24 (1985) 323-326.

[8] D. Bonn, M.M. Denn, Yield stress fluids slowly yield to analysis, Science 324 (2009) 1401-1402.

[9] M. Denn, D. Bonn, Issues in the flow of yield-stress liquids, Rheol. Acta 50 (2011) 307-315. doi: 10.1007/s00397-010-0504-3.

[10] W.H. Herschel, R. Bulkley, Konsistenzmessungen von gummi-benzollösungen, Kolloid-Zeitschrift 39 (1926) 291-300.

[11] W. Herschel, T. Bulkley, Measurement of consistency as applied to rubbery benzene solutions, Am. Soc. Test Proc. 26 (1926) 621-633.

[12] N. Pashias, D.V. Boger, J. Summers, D.J. Glenister, A fifty cent rheometer for yield stress measurement, J. Rheol. 40 (1996) 1179-1189.

[13] N. Roussel, P. Coussot, Â fifty-cent rheometerâ for yield stress measurements: from slump to spreading flow, J. Rheol. 49 (2005) 705-718.

[14] W.R. Schowalter, G. Christensen, Toward a rationalization of the slump test for fresh concrete: comparisons of calculations and experiments, J. Rheol. 42 (1998) 865-870.

[15] Y. Liu, N. Balmforth, S. Hormozi, Axisymmetric viscoplastic dambreaks and the slump test, J. Non Newton Fluid Mech. 258 (2018) 45-57.

[16] N.J. Balmforth, R.V. Craster, A.C. Rust, R. Sassi, Viscoplastic flow over an inclined surface, J. Non Newton Fluid Mech. 142 (2007) 219-243. Viscoplastic fluids: From theory to application.

[17] G. German, V. Bertola, Impact of shear-thinning and yield-stress drops on solid substrates, J. Phys. Condens. Matter 21 (2009)

[18] G. German, V. Bertola, The free-fall of viscoplastic drops, J. Non Newton Fluid Mech. 165 (2010) 825-828.

[19] S. Chen, V. Bertola, Morphology of viscoplastic drop impact on viscoplastic surfaces, Soft Matter 13 (2017) 711-719.

[20] G. German, V. Bertola, The spreading behavior of capillary driven yield-stress drops, Colloids Surf.A 366 (2010) 18-26.

[21] G.G. Stokes, On the theories of the internal friction of fluids in motion, and of the equilibrium and motion of elastic solids, Cambridge Library Collection - Mathematics, Cambridge University Press, 1, 75-129.

[22] A.N. Beris, J.A. Tsamopoulos, R.C. Armstrong, R.A. Brown, Creeping motion of a sphere through a bingham plastic, J. Fluid Mech. 158 (1985) 219?244.

[23] C. Xie, J. Zhang, V. Bertola, M. Wang, Lattice boltzmann modeling for multiphase viscoplastic fluid flow, J. Non Newton Fluid Mech. 234 (2016) 118-128.

[24] A.M.V. Putz, T.I. Burghelea, I.A. Frigaard, D.M. Martinez, Settling of an isolated spherical particle in a yield stress shear thinning fluid., Phys. Fluids (2008) 033102.
[25] Y. Holenberg, O.M. Lavrenteva, U. Shavit, A. Nir, Particle tracking velocimetry and particle image velocimetry study of the slow motion of rough and smooth solid spheres in a yield-stress fluid, Phys. Rev. E 86 (2012) 066301.

[26] M.T. Arigo, G.H. McKinley, An experimental investigation of negative wakes behind spheres settling in a shear-thinning viscoelastic fluid, Rheol. Acta 37 (1998) 307-327.

[27] D. Fraggedakis, Y. Dimakopoulos, J. Tsamopoulos, Yielding the yield-stress analysis: a study focused on the effects of elasticity on the settling of a single spherical particle in simple yield-stress fluids, Soft Matter 12 (2016) 5378-5401.

[28] A.L. Goff, D. Quere, C. Clanet, Shooting in a foam, Soft Matter 10 (2014) 6696-6704

[29] P. Marmottant, F. Graner, An elastic, plastic, viscous model for slow shear of a liquid foam, Eur. Phys. J. E 23 (2007) 337-347.

[30] H. Tabuteau, D. Sikorski, S.J. de Vet, J.R. de Bruyn, Impact of spherical projectiles into a viscoplastic fluid, Phys. Rev. E 84 (2011) 031403.

[31] E. Weber, M. Moyers-Gonzalez, T.I. Burghelea, Thermorheological properties of a carbopol gel under shear, J. Non Newton Fluid Mech. 183-184 (2012) 14-24.

[32] M.M. Gonzalez, T. Burghelea, J. Mak, Linear stability analysis for plane-poiseuille flow of an elastoviscoplastic fluid with internal microstructure for large reynolds numbers, J. Non Newton Fluid Mech. 166 (2011) 515-531.

[33] A. Poumaere, M. Moyers-Gonzalez, C. Castelain, T. Burghelea, Unsteady laminar flows of a carbopol gel in the presence of wall slip, J. Non Newton Fluid Mech. 205 (2014) 28-40.

[34] Z. Kebiche, C. Castelain, T. Burghelea, Experimental investigation of the rayleigh-bénard convection in a yield stress fluid, J. Non Newton Fluid Mech. 203 (2014) 9-23.

[35] R. Sainudiin, M. Moyers-Gonzalez, T. Burghelea, A microscopic gibbs field model for the macroscopic behavior of a viscoplastic fluid, UCDMS Research Report 2014/1, 2014.

[36] R. Sainudiin, M. Moyers-Gonzalez, T. Burghelea, A microscopic gibbs field model for the macroscopic yielding behavior of a viscoplastic fluid, Soft Matter 11 (27) (2015) $5531-5545$.

[37] T. Burghelea, M. Moyers-Gonzalez, R. Sainudiin, A nonlinear dynamical system approach for the yielding behavior of a viscoplastic material, Soft Matter 13 (2017) 2024-2039.

[38] D. Quemada, Rheological modeling of complex fluids: i:the concept of effective volume fraction revisited, Eur. Phys. J. AP (1998) 119-127.

[39] D. Quemada, Rheological modeling of complex fluids: III: dilatant behavior of stabilized suspensions, Eur. Phys. J. AP (1998) 309-320.

[40] D. Quemada, Rheological modeling of complex fluids: IV: thixotropic and "thixoelastic" behavior. start-up and stress relaxation, creep tests and hysteresis cycles, Eur. Phys. J. AP (1999) 191-207.

[41] R.L. Thompson, E.J. Soares, Viscoplastic dimensionless numbers, J. Non Newton Fluid Mech. 238 (2016) 57-64. Viscoplastic Fluids From Theory to Application 2015 (VPF6).

[42] L. Jorgensen, M.L. Merrer, H. Delanoe-Ayari, C. Barentin, Yield stress and elasticity influence on surface tension measurements, Soft Matter 11 (2015) 5111-5121.

[43] T.C. Papanastasiou, Flows of materials with yield, J. Rheol. (1978-present) 31 (1987) 385-404. 